\title{
Measurement-Based Deep Venous Thrombosis Screening System
}

\author{
Julian Guerrero $^{1}$, S.E. Salcudean ${ }^{1}$, James A. McEwen ${ }^{1,2}$, Bassam A. Masri ${ }^{2}$, \\ and Savvas Nicolaou ${ }^{3}$ \\ 1 Department of Electrical \& Computer Engineering, University of British Columbia \\ 2356 Main Mall, Vancouver BC V6T 1Z4, Canada \\ \{juliang,tims\}@ece.ubc.ca, jamc@interchange.ubc.ca \\ 2 Department of Orthopaedics, University of British Columbia \\ 3114 - 910 W 10th Ave, Vancouver BC V5Z 4E3, Canada \\ 3 Department of Radiology, Vancouver Hospital \& Health Sciences Centre \\ 855 West 12th Ave, Vancouver BC V5Z 1M9, Canada
}

\begin{abstract}
An experimental system and interface that indicate the likelihood of deep venous thrombosis using objective measures was developed, based on ultrasound image processing using a modified Star-Kalman algorithm and a sensorized ultrasound probe. Force, location and image data is used to assess a vessel segment for compression. A user interface displays the results using a $3-\mathrm{D}$ representation. A tissue phantom was developed for testing and validation. Initial results with this phantom and healthy volunteers are presented.
\end{abstract}

\section{Introduction}

Deep venous thrombosis (DVT) screening using compression ultrasound (CUS) is a routine procedure currently carried out in many health care facilities. A technician scans a patient to determine whether a thrombus is present in the patient's venous system by performing compression exams (compression-release cycles) at different locations and identifying incompressible vein segments. Failure to detect DVT may cause pulmonary embolism (PE). Overall mortality of untreated DVT has been reported at approximately $2.5 \%$ [ .

These examinations rely on the expertise of the examiner [5]. Screenings may take in excess of 40 minutes and in $76 \%$ of cases require repeat scans 8, increasing overall cost. Therefore, there is a need for a system that can objectively quantify thrombi and their location, through a single examination.

This paper presents our proposed system. A sensorized ultrasound probe is used to acquire data which is processed in order to determine the compressibility of a vein segment by computing two DVT indices. The acquired data is displayed in 3-D through a user interface in an intuitive manner. Preliminary results validating the system are also presented. 


\section{DVT Screening System}

The formation of thrombi in deep veins occurs because of sluggish blood flow or because of a hypercoagulable state. Thrombi may develop on vein walls occluding blood flow, or may break off and flow through the venous system to the lungs, causing a PE.

Currently two methods are generally used for detecting DVT. The first is contrast venography which involves injecting a patient with contrast medium, then obtaining radiographic images of the filling of the veins. This invasive procedure cannot be performed in up to $10 \%$ of patients because of health risks [6]. The second is compression ultrasound, where a technician uses B-mode ultrasound to obtain transverse images of the patient's deep veins, and observes how well they collapse under gentle probe pressure. This is the current method of choice for DVT detection.

\subsection{Compression Ultrasound Examination}

During a CUS examination, a vein is considered free of DVT when it is seen to completely collapse in the transverse plane when force is applied. Otherwise, it is possible that an intraluminal thrombus is present. A thrombus itself cannot be seen directly, since its echogenicity is similar to that of blood.

A complete compression ultrasound screening consists of scanning the deep veins every 2 to $5 \mathrm{~cm}$ from the common femoral vein near the groin down to the deep calf veins, to the extent possible. The results consist of stored 2-D ultrasound images showing compressed and uncompressed vessel segments, and a diagram schematically illustrating the location of thrombi if any were found.

\subsection{Measurement-Based DVT Screening System}

In order to determine the degree of vessel compression, the transverse vessel area must be identified on a series of ultrasound images. The amount of force applied for each of these images permits us to construct a transverse area - applied force relationship for each vessel segment examined, generating an objective measure of the likelihood of DVT.

The characterization of clot compressibility for DVT detection using ultrasound elasticity imaging has previously been reported in [3].

Image Feature Detection. The extraction of the transverse vessel contours is implemented using a modified Star-Kalman algorithm[1]. In the original algorithm the vessel is modeled by a circle. This is not a good model for compressed veins. We assume that a vein contour is accurately described by an ellipse instead. The ellipse radius $r$ is given by

$$
r=\frac{a b}{\sqrt{b^{2} \cos (\theta-\phi)^{2}+a^{2} \sin (\theta-\phi)^{2}}}
$$


where $\theta$ is the eccentric angle, $\phi$ is the angle of the semi-major axis $a$ with respect to the image plane, and $b$ is the semi-minor axis, as shown in Fig. 1(a) Both $r$ and $\theta$ are measured from the center of the ellipse. It is assumed that the ellipse is centered at the origin of the coordinate system.

In the spatial domain we define a state vector $\boldsymbol{x}_{k}=\left[\begin{array}{lll}\boldsymbol{r}_{k} & \boldsymbol{a}_{k} & \boldsymbol{b}_{k} \\ \boldsymbol{\phi}_{k}\end{array}\right]^{\top}$ for each $\theta_{k}=2 \pi k / N$ from $k=1$ to $N$, and write our system as

$$
\boldsymbol{x}_{k+1}=\left[\begin{array}{c}
\frac{\boldsymbol{a}_{k} \boldsymbol{b}_{k}}{\sqrt{\boldsymbol{b}_{k}^{2} \cos ^{2}\left(\theta_{k}-\phi_{k}\right)+\boldsymbol{a}_{k}^{2} \sin ^{2}\left(\theta_{k}-\boldsymbol{\phi}_{k}\right)}} \\
\boldsymbol{a}_{k} \\
\boldsymbol{b}_{k} \\
\boldsymbol{\phi}_{k}
\end{array}\right]+\boldsymbol{\xi}_{k}, \text { and } \boldsymbol{v}_{k}=\boldsymbol{r}_{k}+\boldsymbol{\eta}_{k}(2)
$$

where $\boldsymbol{r}_{k}$ is the radial distance from the ellipse center to its edge along an angle $\theta_{k}$ (see Fig. 1(a)], $\boldsymbol{a}_{k}, \boldsymbol{b}_{k}$ and $\boldsymbol{\phi}_{k}$ are the estimates of $a, b$, and $\phi$ as defined in (1), $\boldsymbol{v}_{k}$ is the measurement vector, and $\boldsymbol{\xi}_{k}$ and $\boldsymbol{\eta}_{k}$ are system and measurement noise sequences, respectively, with known covariances.

The modified Star-Kalman algorithm generates an estimate of the state vector, and the edge locations are described by the estimated $\boldsymbol{r}_{k}$. An ellipse is reconstructed using (1) and the estimated ellipse parameters. A data fit is deemed invalid if an error measure computed from the difference between the estimated points $\boldsymbol{r}_{k}$ and the generated ellipse is larger than a predetermined threshold.

An initial vessel location must be provided, which is subsequently tracked as in 1 .

Location and Force Sensing. The force applied by the probe to the patient is measured by a sensor consisting of two rigid shells with a 6 DOF force-torque sensor (Nano25, ATI Industrial Automation, Inc.) between them, as shown in Figs. 1(b) and 1(c), The examiner grasps the outer shell and manipulates the probe in an ordinary fashion, while applied forces and torques are read into a computer.

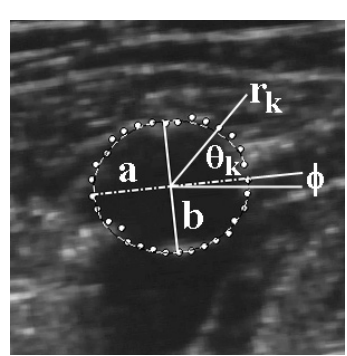

(a)

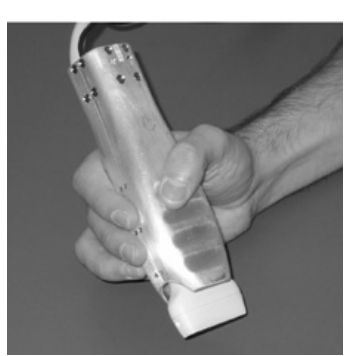

(b)

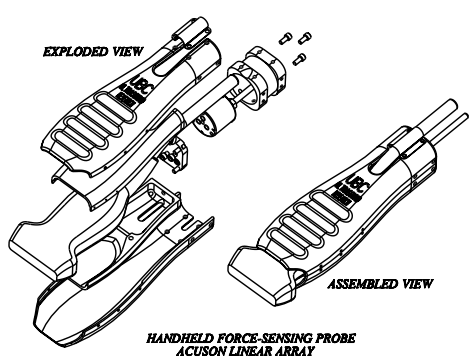

(c)

Fig. 1. (a) Extracted Ellipse, (b) Ultrasound Probe with Shells, and (c) Diagram of Ultrasound Probe Shells 
A 6 DOF electromagnetic location sensor (PCIBird, Ascension Technology Corporation) is attached to the outer shell in order to obtain position and orientation readings. Many systems have used these types of sensors for tracking the location of ultrasound probes [4].

Compression Assessment Criteria. Two distinct measures are used to indicate the possibility of DVT for a vein segment.

The first DVT likelihood measure is called the transverse area ratio (TAR) and is defined as the ratio of the minimum to the maximum transverse vessel area obtained at one vessel section. Values of the transverse areas are approximated by the corresponding extracted ellipse parameters, since the ellipse area is $a b \pi$. A large TAR indicates vein incompressibility and DVT.

The second DVT likelihood measure is obtained by fitting a line to the normalized measurements of area vs. force at the same location. A slope near zero indicates high likelihood of DVT. A slope close to -1 indicates low likelihood of DVT.

Indicators of an adequate examination are also implemented, in order to discard invalid data. These indicators are insufficient data points at the location, insufficient force applied during the compression examination at a location and points with force or area measurements that present large variations with respect to previous measurements.

Data Display. A 3-D polygonal mesh model is constructed from the extracted vessel contours, which is displayed along with a virtual representation of the image plane with the current ultrasound image mapped to its surface. A conventional 2-D ultrasound image is displayed alongside the virtual representation, as shown in Fig. 2.

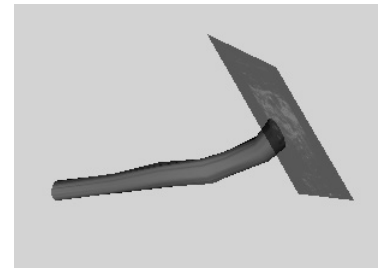

(a)

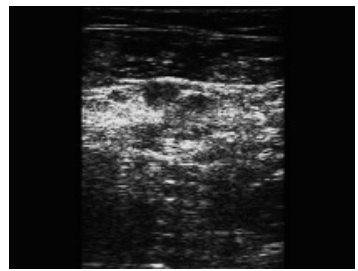

(b)

Fig. 2. Ultrasound Screening System Interface

Compression information for each vessel segment is mapped to the surface of the 3-D vein model using a range of colors. The indicators of exam adequacy are also displayed using color. The examiner can quickly identify from the display which vessel segments have been correctly screened for DVT and which were not, as well as the results from the screening. 


\section{Experimental Results}

\subsection{Feature Detection Results}

The ellipse parameter estimation was verified by generating simulated ultrasound images using Field $\mathrm{II}^{\odot}[7$, with known elliptical features similar to transverse images of a vein. The Star-Kalman estimation was performed on 300 images with different initial conditions and $a, b$ and $\phi$, were estimated to within $4.03 \%$, $7.81 \%$ and $1.79^{\circ}$, respectively, of known ellipse parameters. The computation time was about $300 \mathrm{~ms}$ for each estimation when tested using Matlab ${ }^{\odot}$.

\subsection{Validation of the Transverse Area Ratio (TAR) Criterion}

To validate the TAR criterion, several trials were performed on data obtained from teaching videos. While force measurements were not available for this validation, compression-release cycles were easily identified, as well as the maximum and minimum transverse vessel area.

Examples of partially thrombosed veins $(n=3)$ and of healthy veins $(n=10)$ were identified and processed. The 8 to 20 images for each case were segmented manually by the authors as well as by using the feature extraction algorithm. The average values for the TAR for healthy veins were $0.517 \%$ from the manually segmented data (M) and $12.959 \%$ from the extracted contour (E), while for diseased veins the values were $61.73 \%(\mathrm{M})$ and $59.63 \%(\mathrm{E})$. Fig. 3 shows typical examples of transverse areas of diseased and healthy veins and ellipse area computation.

\subsection{Phantom Experiments}

A phantom was constructed with polyvinyl-alcohol (PVA) cryogel [2] vessels approximately $10 \mathrm{~mm}$ in diameter fixed within a container, as shown in Fig. $4(\mathrm{a})$. The interior of each vessel was accessible from the outside of the Plexiglas container. The tissue phantom surrounding the vessels was made using agar 9 . The vessels were then filled with water to represent a healthy vein or with a $12 \mathrm{~mm} \times 4 \mathrm{~mm}$ cylindrical PVC thrombus mimic and water to represent a thrombosed vein.

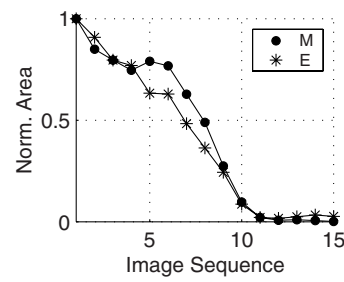

(a)

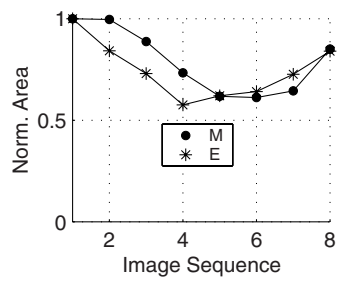

(b)

Fig. 3. Examples of Transverse Vessel Area for (a) Healthy and (b) Diseased Veins 
The phantom vessels were scanned in a transverse plane. Compression-release cycles were performed at several locations, the data was processed, the TAR was computed, and the 3-D model of the vessel was constructed. Fig. 4(b) presents the TAR vs. phantom location for the 'healthy' $(\mathrm{H})$ and 'diseased' (D) case. The location of the thrombus phantom is clearly evident from the high TAR value.

Fig. 4(c) shows a $3-\mathrm{D}$ vein model generated by scanning the healthy vein phantom. The compression information is mapped to the surface of the model as grayscale values. The midsection of the vessel corresponds to a healthy compressible vessel, while both ends which were attached to the fixtures in the phantom show less compressibility. This is also observed in Fig. 4(b)

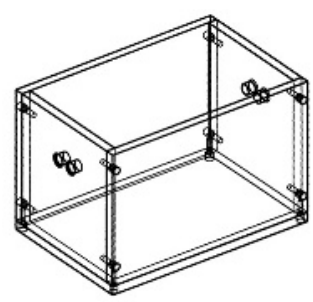

(a)

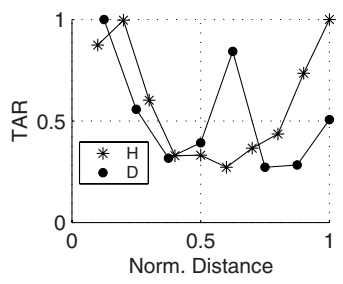

(b)

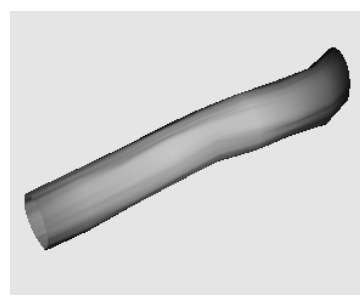

(c)

Fig. 4. (a) Phantom Container (b) Minimum Transverse Vessel Area vs. Location Along Vessel and (c) 3-D Model of Healthy Vein Phantom

\subsection{Healthy Subjects}

Initial testing was performed on healthy volunteers $(n=3)$, and several vessel segments were scanned in each case (total $n=10$ ). No diseased patients were included because of ethical considerations, but will be included for subsequent studies once the system performs reliably.

Typical results of a compression exam on superficial femoral vein are presented in Figs. 5(a) and 5(b), and these indicate the abscence of DVT as expected.

An arterial segment was also examined. The results for one compressionrelease cycle are presented in Fig. 5(c) and clearly indicate an incompressible vessel, as expected.

Invalid data due to a small number of data points at a location was encountered in $15.9 \%$ of cases, due to insufficient force in $12.7 \%$ of cases, and due to outliers in $36.5 \%$ of cases ( $n=63$ locations). This was due to inaccurate tracking because of the low frame-rate of our image acquisition from the ultrasound machine. 


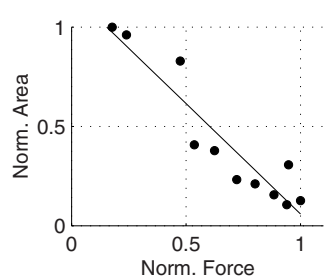

(a)

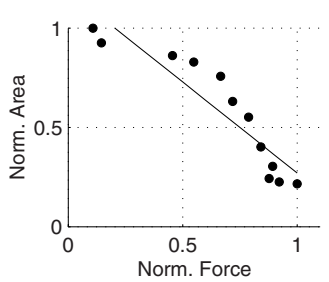

(b)

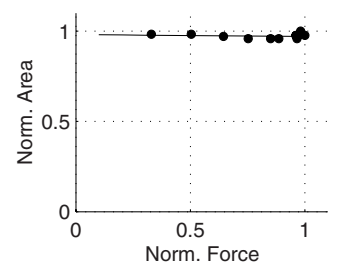

(c)

Fig. 5. Compression Examination Data for (a,b) Veins and (c) Artery

\section{Discussion}

It is clearly evident from the teaching video data that there is a large TAR difference between healthy and diseased veins. The results are consistent, suggesting that DVT can be well characterized by the TAR.

The feature detection algorithm was shown to reliably identify ellipse parameters over a range of conditions, as well as detecting the edge location when tested on images with known features. This performance was also seen when segmenting phantom and human images.

The force and location data provide the information needed to display and store the results of an adequate compression exam. The compressibility and general shape of the examined vessel as well as the relative location of a thrombus, if found, are presented to the user and are archived for future reference.

The results obtained for the examined veins correspond to the expected outcomes. The results obtained from the examined artery illustrate the lack of variation expected from an incompressible vessel, and can be interpreted as a true positive result. It was also shown that the phantom provides an adequate platform for the development and testing of the current system.

The authors are aware that the presence of metals has to be accounted for through calibration of the location sensor. Accuracy errors on known phantoms were found to be small enough not to interfere with the prototype demonstrated in this paper.

\subsection{Future Work}

Many improvements are still possible, as well as in depth testing to properly validate the system.

Improvements to the feature detection algorithm are necessary in order to adequately detect a completely compressed vessel. This inadequate detection is the reason why the data indicates a larger average of the TAR for a healthy vein in Section 3.2. Additionally, bifurcating vessels cannot be tracked with the current system.

The system must be refined in order to improve frame rate and image tracking throughout an examination. It is expected that this will reduce the number of 
invalid datasets during a scan. The decision process for accepting a dataset must also be improved for this purpose.

Extensive clinical and laboratory testing on healthy and diseased subjects is planned for validating the system. The phantom must be validated as well, by insuring that compression vs. area data and ultrasound images obtained from the phantom are consistent with data obtained from humans.

\section{Conclusions}

An experimental system and interface for the screening of DVT has been presented. The system uses automatic area computation, force sensing and sensor location to determine two numerical measures for the likelihood of DVT. These measures along with the sensor data are presented in a 3-D display to the examiner, as well as providing a record of the screening.

The system was evaluated on a custom made phantom as well as on healthy human subjects, with promising results.

\section{References}

1. P. Abolmaesumi, S.E. Salcudean, W.H. Zhu, M.R. Sirouspour, and S.P. DiMaio. Image-Guided Control of a Robot for Medical Ultrasound. IEEE Transactions on Robotics and Automation, 18, 2002.

2. K.C. Chu and B.K. Rutt. Polyvinyl Alcohol Cryogel: An Ideal Phantom Material for MR Studies of Arterial Flow and Elasticity. Magnetic Resonance in Medicine, 37: 314-319, 1997.

3. S.Y. Emelianov, X. Chen, M. O'Donnell, B. Knipp, D. Myers, T.W. Wakefield, and J.M. Rubin. Triplex Ultrasound: Elasticity Imaging to Age Deep Venous Thrombosis. Ultrasound in Medicine and Biology, 28(6): 757-767, 2002.

4. Aaron Fenster and Donal B. Downey. 3-D Ultrasound Imaging: A Review. IEEE Engineering in Medicine and Biology, pages 41-51, November/December 1996.

5. Bradley W. Frazee, Eric R. Snoey, and Andrew Levitt. Emergency Department Compression Ultrasound To Diagnose Proximal Deep Vein Thrombosis. The Journal of Emergency Medicine, 20(2): 107-111, 2001.

6. M.C.H. Janssen, H. Wollersheim, I.R.O. Novakova, F.M.J. Heystraten, W.N.J.C. van Asten, and Th. Thien. Diagnosis Of Deep Vein Thrombosis, An Overview. Netherlands Journal of Medicine, 48: 109-121, 1996.

7. J.A. Jensen. Field: A Program for Simulating Ultrasound Systems. Medical and Biological Engineering and Computing, 34: 351-353, 1996. Supplement 1, Part 1.

8. Nicolas Perone, Henri Bounameaux, and Arnaud Perrier. Comparison Of Four Strategies For Diagnosing Deep Vein Thrombosis: A Cost-Effectiveness Analysis. The American Journal of Medicine, 110(1): 33-40, January 2001.

9. D.W. Rickey, P.A. Picot, D.A. Christopher, and A. Fenster. A Wall-less Vessel Phantom for Doppler Ultrasound Studies. Ultrasound in Medicine and Biology, 21(9): 1163-1176, 1995. 